\title{
Recent Trends in Research on Semiconductor Nanostructures for Lasers, Optics and Photonics Applications
}

\section{Navendu Goswami}

Department of Physics and Material Science \& Engineering, Jaypee Institute of Information Technology, UP, India

"Corresponding author: Navendu Goswami, Assistant Professor (Senior Grade), Department of Physics and Material Science \& Engineering, Jaypee Institute of Information Technology, UP, India, Tel:+91-120-2594364 (O); Fax:+91-120-2400986; E-mail:navendugoswami@gmail.com

Rec date: June 04, 2014; Acc date: June 05, 2014; Pub date: June 11, 2014

Copyright: @ 2014 Goswami N. This is an open-access article distributed under the terms of the Creative Commons Attribution License, which permits unrestricted use, distribution, and reproduction in any medium, provided the original author and source are credited.

Citation: Navendu Goswami (2014) Recent Trends in Research on Semiconductor Nanostructures for Lasers, Optics and Photonics Applications. J Laser Opt Photonics 1:e102. doi: 10.4172/jlop.1000e102

\section{Editorial}

As we are moving in the second decade of this twenty-first century, it is crucial to notice the vast changing era of scientific community that has advanced a lot through semiconducting technologies of microelectronic storage devices, sensing, true vision optical displays devices and also a whole area of telecommunications, information security etc. that has completely revolutionized the daily lifestyle. The building blocks of this revolution, generally referred as information revolution, were laid in last century itself and its impact and significance is enhancing with each passing day of present era.

It is important here to note that semiconductors are quite different from either metals or insulators, and their significance lies in the foundation they provide for a massive microelectronics and optics industries. The advent of Nanotechnology spurred the futuristic applications of the semiconductors materials in optoelectronic fields such as lasers for telecommunications, information processing, remote sensing, materials processing, and as a potent diagnostic tool. It is therefore crucial to publish recent research progress in this specific field so as to address and highlight the challenges pertinent to complementary local (nanoscale) and more global (micro- or macroscale) experimental methods to characterize the nanoscale semiconductors. The field of spintronics has also emerged as a promising source of new technologies that will help to meet the needs of information and communications industries. The segment of research in this domain would reflect the recent trends in spintronics research and could also provide a good overview of the hitherto status of investigations into spin-orbit interaction and its effect on charge transport.

Recently, plasmonics, an area of research in nanophotonics, is attracting increasing interest due to its potential applications in sensing and detecting, sub-wavelength confinement of light and integrated circuits etc. In particular, when plasmonic structures such as metal nanostructures or highly doped semiconductor particles are combined with active semiconductor materials and nanostructures, novel exciting physics and applications arise. Also, the integration of III-V semiconductor devices with silicon is one of the most topical challenges in current electronic materials research. The combination has the potential to exploit the unique optical and electronic functionality of III-V technology with the signal processing capabilities and advanced low-cost volume production techniques associated with silicon. Semiconductor nanostructures that are of current interest for the above mentioned applications are Si based undoped and highly metal doped semiconductors and in the past two decades, there has been increasing research and industrial activities in the area of Gallium
Nitride (GaN) electronics, stimulated first by the successful demonstration of GaN LEDs. The GaN/GaAlN electronics is a rapidly evolving research area worldwide, the reviews and research articles may cover the topics of growth and heteroepitaxy, bulk GaN substrates, theory and modelling, optical properties, laser diodes and LEDs, as well as transport properties and GaN/GaAlN based electronics. Nanostructures in general, are considered as the basic building blocks for the next generation of electronics, photonics, sensors and energy applications. It is undoubtedly important that the synthesis and growth of semiconductor nanostructures of a broad range of materials should be promptly reported so as to keep track of novel techniques to synthesize technologically potent metal-oxide nanostructures of $\mathrm{ZnO}, \mathrm{CuO}, \mathrm{TiO}_{2}$ etc. for lasers, optics and photonic applications.

It is more than fifty years now since first semiconductor diode laser was reported and hence it is high time to report research and review articles charting the latest developments in semiconductor laser technology. The concept of the double heterostructure and its efficacy for semiconductor lasers was proposed first in 1962. Femtosecond sources have been used to monitor the course of chemical reactions, to observe molecular dynamics, to perform 3-D imaging of biological tissue, and to study carrier dynamics in semiconductors and for ultrahigh-speed optoelectronics. Articles and issues on recent progress and the futuristic opportunities in the exciting field of lasers could be based on the research and development of sub-100 femtosecond pulses, which is the current area of curiosity. Novel or improved sources of ultra-short pulses are under development, including high-power fibrebased lasers. Nonlinear propagation effects with femtosecond pulses are also the subject of extensive research, including the generation of spatio-temporal solutions (i.e., pulses propagated without diffracting in space or dispersing in time) fibre delivery of high power pulses, and laser filaments in condensed matter and gases. On the other hand, solid-state organic amplifiers and lasers are attractive for hybrid integration due to their compatibility with different material platforms, straightforward processing, and possibility to easily optimize their optical and electronic properties via molecular engineering. In this context, it is necessary to focus on gain medium designs and synthesis in conjunction with new resonator architectures that may finally lead to tremendous improvements in temporal and spectral properties, lifetime stability, gains produced and operating threshold powers; which shall directly trigger their use for vast range of integrated photonic applications.

A recent surge in optics research worldwide is devoted to Nanophotonics that signifies the utility of nanostructures to enhance the interaction of light with matter for applications such as, ultrahigh 
Citation: Navendu Goswami (2014) Recent Trends in Research on Semiconductor Nanostructures for Lasers, Optics and Photonics Applications. J Laser Opt Photonics 1: e102. doi:10.4172/2469-410X.1000e102

Page 2 of 2

speed all-optical processing, frequency metrology, novel mid-infrared sources, and quantum information technology etc. Semiconductor quantum dots and defects are being investigated for potential development of highly broadband optical amplifiers, solar cells, and quantum computing and information processing. In addition, nonlinear optical processes in silicon nanostructures are also being probed to produce devices that can be used for high precision alloptical clocks and highly compact sources for mid-infrared optical spectroscopy. In present time, optical technologies are expanding into literally all areas of modern life. Already, over 90 percent of all the data transferred for the telecommunication is transmitted through optical fibres. In microelectronics, storage media and microprocessors are produced using optical techniques. Medicine, too, is increasingly relying on optical technologies, for both diagnosis and therapy. In production engineering, laser techniques are systematically gaining ground in metrology and manufacturing. High productivity, flexibility and quality are the key advantages of laser technology Researchers are engaged in various cutting-edge research efforts in optics for example, 3-D biomedical imaging and controlling individual solid-state spins at the nanometric scale.

We are confident that the breadth and quality of articles to be published in this journal would make it an excellent standard reference for beginner as well as experienced researchers active in the field of synthesis and characterization of diverse semiconductor nanostructures and subsequently their applications for Lasers, Optics and Photonics. The articles that address aforesaid purpose are worthy of publication and we look forward to provide an excellent updated spectrum of promising research activities on semiconductor nanostructures through Journal of Lasers, Optics \& Photonics. 